\title{
APPROXIMATIONS OF SOLUTIONS TO A RETARDED TYPE FRACTIONAL DIFFERENTIAL EQUATION WITH A DEVIATED ARGUMENT
}

\author{
PRADEEP KUMAR, D.N. PANDEY AND D. BAHUGUNA \\ Communicated by Neville Ford
}

\begin{abstract}
In the present work, we are concerned with approximations of solutions to a retarded type fractional differential equation with a deviated argument in a separable Hilbert space $H$. We consider an integral equation associated with a given problem and then consider a sequence of approximate integral equations. We prove the existence, uniqueness and convergence to each of the approximate integral equations by using analytic semigroup theory and the fixed point method. We also prove that the limiting function satisfies the associated integral equation. Finally, we consider Faedo-Galerkin approximations of solutions and prove some convergence results.
\end{abstract}

1. Introduction. In this article, we consider the following retarded type fractional differential equation with a deviated argument in a separable Hilbert space $(H,\|\cdot\|,(\cdot, \cdot))$ :

$$
\left.\begin{array}{lr}
{ }^{c} D_{t}^{\eta}[u(t)+g(t, u(t))]+A u(t)=f(t, u(t), u[h(u(t), t)]), & \eta \in[0,1), \\
u(0)=u_{0}, & 0<t \leq T<\infty
\end{array}\right\}
$$

where ${ }^{c} D_{t}^{\eta}$ is the Caputo fractional derivative of order $\eta$ and $A: D(A) \subset$ $H \rightarrow H$ is a closed, densely defined, positive definite, self-adjoint linear operator which satisfies assumption (H1), stated later. Functions $f, g$

2010 AMS Mathematics subject classification. Primary 34G10, 34G20, 34K30, $35 \mathrm{~K} 90,47 \mathrm{~N} 20$.

Keywords and phrases. Analytic semigroup, retarded type fractional differential equation, deviated argument, Banach fixed point theorem, Faedo-Galerkin approximation.

The first author is the corresponding author.

The third author would like to acknowledge financial aid from the Department of Science and Technology, New Delhi, under its research project SR/S4/MS:796/12.

Received by the editors on February 21, 2013, and in revised form on July 18, 2013. 
and $h$ are suitably defined and satisfy certain conditions to be stated later.

In the present work, we are interested in Faedo-Galerkin approximations of solutions to problem (1.1). In [20], Milleta has discussed the Faedo-Galerkin approximations of solutions to the particular case of (1.1) in the cases when $\eta=1, h \equiv 0$ and $f(t, u)=M(u)$. For a nice introduction and related study of various problems in this direction, we refer to the reader to $[\mathbf{1}, \mathbf{2}, \mathbf{3}, \mathbf{4}, \mathbf{2 0}, \mathbf{2 2}, \mathbf{2 3}]$ and the references cited therein.

In [23], Muslim et al. have established the existence, uniqueness and convergence of approximations of solutions in a separable Hilbert space and convergence of the Faedo-Galerkin approximations of solutions to the following problem:

$$
\begin{aligned}
u(t)=u_{0} & +\frac{1}{\Gamma \beta} \int_{0}^{t}(t-s)^{\beta-1}(-A u(s)) d s \\
& +\frac{1}{\Gamma \beta} \int_{0}^{t}(t-s)^{\beta-1} f(s, u(s), u(a(s))) d s,
\end{aligned}
$$

where $-A$ is the infinitesimal generator of an analytic semigroup of bounded linear operators $\{S(t), t \geq 0\}$ on a Banach space $(H,\|\cdot\|,(\cdot, \cdot))$, the functions $f:[T] \times H \times H \rightarrow H$ and $a:[0, T] \rightarrow[0, T]$ are suitable functions.

In [24], Ntouyas et al. proved existence results for semilinear neutral functional differential inclusions with finite or infinite delay in Banach spaces to the following problem

$\frac{d}{d t}\left[y(t)-f\left(t, y_{t}\right)\right]=A y(t)+F\left(t, y_{t}\right)$, almost everywhere $t \in J:=[0, T]$,

$$
y(t)=\Phi(t), \quad t \in J_{0}:=[-r, 0],
$$

where $f: J \times D \rightarrow E, F: J \times D \rightarrow \mathcal{P}(E)$ is a multivalued map, $\mathcal{D}=\{\Psi:[-r, 0] \rightarrow E: \Psi$ is continuous $\}, \Phi \in \mathcal{D}, 0<r<\infty, E$ is a real separable Banach space with norm $\|\cdot\|$ and $\mathcal{P}(E)$ is the family of all nonempty subsets of $E$.

For earlier work on the existence and uniqueness of solutions to differential equations of fractional order, we refer to $[\mathbf{5}, \mathbf{1 2}, \mathbf{1 4}, \mathbf{1 5}$, 
16, 17, 18, 19, 21, 27, 28, 31, 33] and the references cited therein.

The book [6] by El'sgol'ts and Norkin provides a comprehensive study of differential equations with deviated arguments. The existence, uniqueness, almost automorphic solutions and asymptotic behaviors of differential equations with deviating arguments has been studied by many authors like Grimm [8], Obreg [25], Driver [10], Gal [7] (see also $[\mathbf{9}, \mathbf{1 1}, \mathbf{1 3}, \mathbf{2 9}, \mathbf{3 2}])$ and the references cited therein.

The rest of the paper is organized as follows. In Section 2, we put some notations, notions and results that are required for proving the main results. In Section 3, we consider an integral equation associated with problem (1.1) and then consider a sequence of approximate integral equations and establish the existence and uniqueness of solutions to each of the approximate integral equations. We also prove the convergence of solutions to each of the approximate integral equations in Section 4 and then prove that the limiting function satisfies the associated integral equation. In Section 5, we consider the Faedo-Galerkin approximations of solutions and prove some convergence results for such approximations. Finally, we give an example to demonstrate the applications of abstract results obtained in the earlier sections.

2. Preliminaries and assumptions. In this section, we present some assumptions, preliminaries and lemmas required for proving the main results. The details of the material presented here can be found in [26]. We shall use the following assumption on operator $A$ :

(H1) Let $A$ be a closed, positive definite, self-adjoint linear operator from the domain $D(A) \subset H$ into $H$ with $D(A)$ dense in $H$. We also assume that $A$ has the pure point spectrum

$$
0<\lambda_{0} \leq \lambda_{1} \leq \lambda_{2} \leq \cdots \leq \lambda_{m} \leq \cdots,
$$

where $\lambda_{m} \rightarrow \infty$ as $m \rightarrow \infty$ and a corresponding complete orthonormal system of eigenfunctions $\left\{\phi_{i}\right\}$, i.e.,

$$
A \phi_{i}=\lambda_{i} \phi_{i} \quad \text { and } \quad\left\langle\phi_{i}, \phi_{j}\right\rangle=\delta_{i j}
$$

where $\delta_{i j}=1$ for $i=j$, zero otherwise.

Assumption (H1) implies that $-A$ generates an analytic semigroup of bounded linear operators $S(t), t \geq 0$. Then there exist constants $\widetilde{M} \geq 1$ 
and $\omega \geq 0$ such that

$$
\|S(t)\| \leq \widetilde{M} e^{\omega t}, \quad t \geq 0 .
$$

We also note that [26, Lemma 4.2, page 52]

$$
\left\|\frac{d^{i}}{d t^{i}} S(t)\right\| \leq M_{i}, \quad t>t_{0}
$$

for some positive constant $M_{i}$.

Without loss of generality, we may assume that $\|S(t)\|$ is uniformly bounded by $M$, i.e., $\|S(t)\| \leq M$ for $t \geq 0$, and that $0 \in \rho(-A)$, i.e., $-A$ is invertible. This allows us to define the positive fractional power $A^{\alpha}$ for $0 \leq \alpha \leq 1$ as closed linear operator with domain $D\left(A^{\alpha}\right) \subseteq H$. Furthermore, $D\left(A^{\alpha}\right)$ is dense in $H$ endowed with the norm

$$
\|x\|_{\alpha}=\left\|A^{\alpha} x\right\| .
$$

Henceforth, we denote the space $D\left(A^{\alpha}\right)$ by $H_{\alpha}$ endowed with the norm $\|\cdot\|_{\alpha}$. Also, for each $\alpha>0$, we define $H_{-\alpha}=\left(H_{\alpha}\right)^{*}$, the dual space of $H_{\alpha}$ endowed with the norm $\|x\|_{-\alpha}=\left\|A^{-\alpha} x\right\|$.

Lemma 2.1 ([26, pages 72, 74, 195-196]). Suppose that $-A$ is the infinitesimal generator of an analytic semigroup $S(t), t \geq 0$ with $\|S(t)\| \leq M$ for $t \geq 0$ and $0 \in \rho(-A)$. Then

(i) $H_{\alpha}$ is a Hilbert space for $0 \leq \alpha \leq 1$;

(ii) for any $0<\delta \leq \alpha$ implies $D\left(A^{\alpha}\right) \subset D\left(A^{\delta}\right)$, the embedding $H_{\alpha} \hookrightarrow H_{\delta}$ is continuous;

(iii) the operator $A^{\alpha} S(t)$ is bounded for every $t>0$ and

$$
\left\|A^{\alpha} S(t)\right\| \leq C_{\alpha} t^{-\alpha} .
$$

We denote the space of all $H_{\alpha}$-valued continuous functions on $[0, t]$ by $\mathcal{C}_{t}^{\alpha}=C\left([0, t] ; H_{\alpha}\right)$, for all $t \in(0, T]$. Then $\mathcal{C}_{t}^{\alpha}$ is a Banach space endowed with the norm,

$$
\|\psi\|_{t, \alpha}:=\sup _{0 \leq r \leq t}\|\psi(r)\|_{\alpha}, \quad \psi \in \mathcal{C}_{t}^{\alpha} .
$$

For $0 \leq \alpha<1$, define

$$
\mathcal{C}_{T}^{\alpha-1}=\left\{y \in \mathcal{C}_{T}^{\alpha}:\|y(t)-y(s)\|_{\alpha-1} \leq L|t-s|, \quad \forall t, s \in[0, T]\right\},
$$


where $L$ is a suitable positive constant to be specified later.

We assume the following conditions:

(H2) Let $U_{1} \subset \operatorname{Dom}(f)$ be an open subset of $\mathbf{R}_{+} \times H_{\alpha} \times H_{\alpha-1}$ and, for each $(t, u, v) \in U_{1}$, there is a neighborhood $V_{1} \subset U_{1}$ of $(t, u, v)$. The nonlinear map $f: \mathbf{R}_{+} \times H_{\alpha} \times H_{\alpha-1} \rightarrow H$ satisfies the following condition:

$$
\|f(t, x, \psi)-f(s, y, \widetilde{\psi})\| \leq L_{f}\left[|t-s|^{\theta_{1}}+\|x-y\|_{\alpha}+\|\psi-\widetilde{\psi}\|_{\alpha-1}\right],
$$

where $0<\theta_{1} \leq 1,0 \leq \alpha<1, L_{f}>0$ is a constant, $(t, x, \psi) \in V_{1}$ and $(s, y, \widetilde{\psi}) \in V_{1}$.

(H3) Let $U_{2} \subset \operatorname{Dom}(h)$ be an open subset of $H_{\alpha} \times \mathbf{R}_{+}$and, for each $(x, t) \in U_{2}$, there is a neighborhood $V_{2} \subset U_{2}$ of $(x, t)$. The map $h: H_{\alpha} \times[0, T] \rightarrow[0, T]$ satisfies the following condition:

$$
|h(x, t)-h(y, s)| \leq L_{h}\left[\|x-y\|_{\alpha}+|t-s|^{\theta_{2}}\right],
$$

where $0<\theta_{2} \leq 1,0 \leq \alpha<1, L_{h}>0$ is a constant, $(x, t),(y, s) \in V_{2}$ and $h(\cdot, 0)=0$.

(H4) Let $U_{3} \subset \operatorname{Dom}(g)$ be an open subset of $[0, T] \times H_{\alpha-1}$ and, for each $(t, x) \in U_{3}$, there is a neighborhood $V_{3} \subset U_{3}$ of $(x, t)$. There exists a positive constant $\beta, 0<\alpha<\beta<1$, such that the function $A^{\beta} g$ is continuous for $(t, u) \in\left[0, T_{0}\right] \times H_{\alpha-1}$ such that

$$
\left\|A^{\beta} g(t, x)-A^{\beta} g(s, y)\right\| \leq L_{g}\left\{|t-s|+\|x-y\|_{\alpha-1}\right\},
$$

and

$$
L_{g}\left\|A^{\alpha-\beta-1}\right\| \leq \delta<1
$$

where $L_{g}, \delta>0$ is a positive constant and $(x, t),(y, s) \in V_{3}$.

3. Approximate integral equations. The existence of a solution to (1.1) is closely related to the following integral equation (3.9).

Definition 1 ([31, Definition 1.2]). Let $f \in L^{1}((0, T), H)$ and $\alpha \geq 0$. Then the expression

$$
\begin{gathered}
I_{t}^{\alpha} f(t)=\left(f * \Theta_{\alpha}\right)(t)=\frac{1}{\Gamma \alpha} \int_{0}^{t}(t-s)^{\alpha-1} f(s) d s \\
t>0, \quad \alpha>0
\end{gathered}
$$


where $I_{t}^{0} f(t)=f(t)$ and

$$
\Theta_{\alpha}(t)=\left\{\begin{aligned}
\frac{1}{\Gamma \alpha} t^{\alpha-1}, & t>0 \\
0, & t \leq 0
\end{aligned}\right.
$$

and $\Theta_{0}(t)=0$, is called the Riemann-Liouville integral of order $\alpha$ of $f$.

Definition 2 ([31, Definition 1.3]). Let $f \in C^{m-1}((0, T), H),\left(\Theta_{m-\alpha} *\right.$ $f) \in W^{m, 1}((0, T), H)(m \in \mathbf{N}, 0 \leq m-1<\alpha<m)$. Then the expression

$$
{ }^{c} D_{t}^{\alpha} f(t)=D_{t}^{m} I_{t}^{m-\alpha}\left(f(t)-\sum_{0}^{m-1} f^{i}(0) \Theta_{i+1}(t)\right),
$$

where $D_{t}^{m}=d^{m} /\left(d t^{m}\right)$, is called the Caputo fractional derivative of order $\alpha$ of $f$.

Then, by definitions (1) and (3.2), we can rewrite (1.1) as

$$
\begin{aligned}
u(t)= & \left(u_{0}+g\left(0, u_{0}\right)\right)-g(t, u(t)) \\
& -\frac{1}{\Gamma \alpha} \int_{0}^{t}(t-s)^{\alpha-1}[A u(s)+g(s, u(s))] d s \\
& +\frac{1}{\Gamma \alpha} \int_{0}^{t}(t-s)^{\alpha-1} f(s, u(s), u(h(u(s), s))) d s, \quad t \in[0, T]
\end{aligned}
$$

For a fixed $R>0$, we choose $0<T_{0}=T_{0}\left(\alpha, \beta, u_{0}\right) \leq T$ sufficiently small, such that

$$
C_{\alpha+1-\beta} L_{g}\left\|A^{-1}\right\| \frac{T_{0}^{\eta(\beta-\alpha)}}{\beta-\alpha}+C_{\alpha} L_{f}\left[2+L L_{h}\right] \frac{T_{0}^{\eta(1-\alpha)}}{1-\alpha} \leq 1-\delta,
$$

where $\delta=L_{g}\left\|A^{\alpha-\beta-1}\right\|<1$ and $T_{0}<\min \left(d_{1}, d_{2}\right)$ with

$$
\begin{aligned}
& d_{1}=\left(\frac{R}{4}(\beta-\alpha)\left(C_{1+\alpha-\beta} L_{g}\right)^{-1}\right)^{1 /[\eta(\beta-\alpha)]} \\
& d_{2}=\left(\frac{R}{4}(1-\alpha)\left(C_{\alpha}\left[2+L L_{h}\right] L_{f}\right)^{-1}\right)^{1 /[\eta(1-\alpha)]}
\end{aligned}
$$


and satisfying the following

$$
\left\|\left(S\left(t^{\eta} \theta\right)-I\right) A^{\alpha}\left[u_{0}+g_{n}\left(0, u_{0}\right)\right]\right\|+\left\|A^{\alpha-\beta}\right\| L_{g}\left[T_{0}+\left\|A^{-1}\right\| R\right] \leq \frac{R}{2}
$$

for all $t \in\left[0, T_{0}\right]$ and

$$
C_{\alpha+1-\beta} N_{1} \frac{T_{0}^{\eta(\beta-\alpha)}}{\beta-\alpha}+C_{\alpha} N \frac{T_{0}^{\eta(1-\alpha)}}{1-\alpha} \leq \frac{R}{2} .
$$

For more details of choosing such a $T_{0}$, we refer to [7, Theorem 2.2].

We set

$$
\mathcal{W}=\left\{u \in \mathcal{C}_{T_{0}}^{\alpha} \cap \mathcal{C}_{T_{0}}^{\alpha-1}: u(0)=u_{0}, \quad\left\|u-u_{0}\right\|_{T_{0}, \alpha} \leq R\right\}
$$

Clearly, $\mathcal{W}$ is a closed, bounded subset of $\mathcal{C}_{T_{0}}^{\alpha-1}$ and complete.

Definition 3 ([5, page 434]). By a solution of problem (1.1), we mean a function $u:[0, T] \rightarrow H_{\alpha}$ satisfying the following three conditions:

(i) $u(\cdot)+g(\cdot, u(\cdot)) \in \mathcal{C}_{T}^{\alpha-1} \cap C([0, T], H)$.

(ii) $u(t)+g(t, u(t)) \in D(A)$ and $(t, u(t), u[h(u(t), t)]) \in U_{1}$ for all $t \in[0, T]$.

(iii) $d^{\eta} / d t^{\eta}[u(t)+g(t, u(t))]+A u(t)=f(t, u(t), u[h(u(t), t)])$ for all $t \in(0, T]$.

(iv) $u(0)=u_{0}$.

Definition 4 ([30, Definition 2.7]). By the mild solution of Cauchy problem (1.1), we mean a continuous function $u:\left(0, T_{0}\right] \rightarrow H$ which satisfies the following integral equation associated with (1.1):

$$
\begin{aligned}
u(t)= & \int_{0}^{\infty} \theta \xi_{\eta}(\theta) S\left(t^{\eta} \theta\right)\left[u(0)+g\left(0, u_{0}\right)\right] d \theta-g(t, u(t)) \\
& +\eta \int_{0}^{t} \int_{0}^{\infty} \theta \xi_{\eta}(\theta)(t-s)^{\eta-1} A S\left((t-s)^{\eta} \theta\right) g(s, u(s)) d \theta d s \\
& +\eta \int_{0}^{t} \int_{0}^{\infty} \theta \xi_{\eta}(\theta)(t-s)^{\eta-1} S\left((t-s)^{\eta} \theta\right) \\
& \times f(s, u(s), u[h(u(s), s)]) d \theta d s, \quad t \in\left(0, T_{0}\right] .
\end{aligned}
$$


where

$$
\begin{gathered}
\xi_{\eta}(\theta)=\frac{1}{\eta} \theta^{-1-(1 / \eta)} \rho_{\eta}\left(\theta^{-1 / \eta}\right) \geq 0 \\
\rho_{\eta}(\theta)=\frac{1}{\pi} \sum_{n=1}^{\infty}(-1)^{n-1} \theta^{-n \eta-1} \frac{\Gamma(n \eta+1)}{n !} \sin (n \pi \eta), \quad \theta \in(0, \infty),
\end{gathered}
$$

$\xi_{\eta}$ is a probability density function defined on $(0, \infty)$, that is,

$$
\int_{0}^{\infty} \xi_{\eta}(\theta) d \theta=1
$$

Also, we have

$$
\int_{0}^{\infty} \theta^{\gamma} \xi_{\eta}(\theta)=\int_{0}^{\infty} \frac{1}{\theta^{\gamma \beta}} \rho_{\eta}(\theta)=\frac{\Gamma(1+\gamma)}{\Gamma(1+\gamma \eta)}, \quad \text { for any } \gamma \in[0,1] .
$$

For more details, we refer to $[\mathbf{5}, \mathbf{3 1}, \mathbf{3 3}]$.

Let $H_{n} \subseteq H$ denote the finite dimensional subspace spanned by $\left\{u_{0}, u_{1}, \cdots, u_{n}\right\}$, and let $P^{n}: H \rightarrow H_{n}$ be the corresponding projection operator for $n=0,1,2, \ldots$ We define

$$
\begin{aligned}
& g_{n}: \mathbf{R}_{+} \times H \longrightarrow H \quad \text { as } \quad g_{n}(t, u(t))=g\left(t, P^{n} u(t)\right) \quad \text { and } \\
& f_{n}: \mathbf{R}_{+} \times H \times H \longrightarrow H \quad \text { given by } \\
& f_{n}(s, u(s), u[h(u(s), s)])=f\left(s, P^{n} u(s), P^{n} u[h(u(s), s)]\right) .
\end{aligned}
$$

For $n=0,1, \ldots$, we define a map $\mathcal{F}_{n}: \mathcal{W} \rightarrow \mathcal{W}$ given by

$$
\begin{aligned}
&\left(\mathcal{F}_{n} u\right)(t)= \int_{0}^{\infty} \theta \xi_{\eta}(\theta) S\left(t^{\eta} \theta\right)\left[u(0)+g_{n}\left(0, u_{0}\right)\right] d \theta-g_{n}(t, u(t)) \\
&+\eta \int_{0}^{t} \int_{0}^{\infty} \theta \xi_{\eta}(\theta)(t-s)^{\eta-1} A S\left((t-s)^{\eta} \theta\right) g_{n}(s, u(s)) d \theta d s \\
&+\eta \int_{0}^{t} \int_{0}^{\infty} \theta \xi_{\eta}(\theta)(t-s)^{\eta-1} S\left((t-s)^{\eta} \theta\right) \\
&(3.12) \quad \times f_{n}(s, u(s), u[h(u(s), s)]) d \theta d s, \quad t \in\left(0, T_{0}\right] .
\end{aligned}
$$

Theorem 3.1. Let assumptions (H1)-(H4) hold and also let $u_{0} \in H_{\alpha}$ for $0 \leq \alpha<1$. Then there exists a unique $u_{n} \in \mathcal{C}_{T_{0}}^{\alpha-1} \cap \mathcal{C}_{T_{0}}^{\alpha}$ such that $\mathcal{F}_{n} u_{n}=u_{n}$ for each $n=0,1,2, \ldots, u_{n}$ satisfies the following 
approximate integral equation corresponding to the integral equation $(3.9)$,

$$
\begin{aligned}
& u_{n}(t)= \int_{0}^{\infty} \theta \xi_{\eta}(\theta) S\left(t^{\eta} \theta\right)\left[u(0)+g_{n}\left(0, u_{0}\right)\right] d \theta-g_{n}\left(t, u_{n}(t)\right) \\
&+\eta \int_{0}^{t} \int_{0}^{\infty} \theta \xi_{\eta}(\theta)(t-s)^{\eta-1} A S\left((t-s)^{\eta} \theta\right) g_{n}\left(s, u_{n}(s)\right) d \theta d s \\
&+\eta \int_{0}^{t} \int_{0}^{\infty} \theta \xi_{\eta}(\theta)(t-s)^{\eta-1} S\left((t-s)^{\eta} \theta\right) \\
&(3.13) \quad \times f_{n}\left(s, u_{n}(s), u\left[h\left(u_{n}(s), s\right)\right]\right) d \theta d s, \quad t \in\left(0, T_{0}\right] .
\end{aligned}
$$

Proof. In order to prove this theorem, first we need to show that $\mathcal{F}_{n} u \in \mathcal{C}_{T_{0}}^{\alpha-1}$ for any $u \in \mathcal{C}_{T_{0}}^{\alpha-1}$. Clearly, $\mathcal{F}_{n}: \mathcal{C}_{T_{0}}^{\alpha} \rightarrow \mathcal{C}_{T_{0}}^{\alpha}$.

If $u \in \mathcal{C}_{T_{0}}^{\alpha-1}, T_{0}>t_{2}>t_{1}>0$, and $0 \leq \alpha<1$, then we get

$$
\begin{aligned}
&\left\|\left(\mathcal{F}_{n} u\right)\left(t_{2}\right)-\left(\mathcal{F}_{n} u\right)\left(t_{1}\right)\right\|_{\alpha-1} \\
& \leq \int_{0}^{\infty} \theta \xi_{\eta}(\theta)\left\|\left(S\left(t_{2}^{\eta} \theta\right)-S\left(t_{1}^{\eta} \theta\right)\right)\left(u_{0}+g_{n}\left(0, u_{0}\right)\right)\right\|_{\alpha-1} d \theta \\
&+\left\|A^{\alpha-1-\beta}\right\|\left\|A^{\beta} g_{n}\left(t_{2}, u\left(t_{2}\right)\right)-A^{\beta} g_{n}\left(t_{1}, u\left(t_{1}\right)\right)\right\| \\
&+\int_{0}^{t_{1}} \int_{0}^{\infty} \xi_{\eta}(\theta) \\
&\left\|\left(\eta \theta\left(t_{2}-s\right)^{\eta-1} A S\left(\left(t_{2}-s\right)^{\eta} \theta\right)-\eta \theta\left(t_{1}-s\right)^{\eta-1} A S\left(\left(t_{1}-s\right)^{\eta} \theta\right)\right)\right\| \\
& \times\left\|A^{\alpha-1} g_{n}(s, u(s))\right\| d \theta d s \\
&+\int_{t_{1}}^{t_{2}} \int_{0}^{\infty} \xi_{\eta}(\theta)\left\|\eta \theta\left(t_{2}-s\right)^{\eta-1} A S\left(\left(t_{2}-s\right)^{\eta} \theta\right)\right\| \\
&\left\|A^{\alpha-1} g_{n}(s, u(s))\right\| d \theta d s \\
&+\int_{0}^{t_{1}} \int_{0}^{\infty} \xi_{\eta}(\theta) \|\left(\eta \theta\left(t_{2}-s\right)^{\eta-1} S\left(\left(t_{2}-s\right)^{\eta} \theta\right)-\eta \theta\left(t_{1}-s\right)^{\eta-1}\right. \\
&\left.S\left(\left(t_{1}-s\right)^{\eta} \theta\right)\right) A^{\alpha-1}\|\| f_{n}(s, u(s), u[h(u(s), s)]) \| d \theta d s \\
&+\int_{t_{1}}^{t_{2}} \int_{0}^{\infty} \xi_{\eta}(\theta)\left\|\eta \theta\left(t_{2}-s\right)^{\eta-1} S\left(\left(t_{2}-s\right)^{\eta} \theta\right) A^{\alpha-1}\right\|
\end{aligned}
$$




$$
\left\|f_{n}(s, u(s), u[h(u(s), s)])\right\| d \theta d s
$$

For the first part of the right hand side of (3.14), we have

$$
\begin{aligned}
& \int_{0}^{\infty} \xi_{\eta}(\theta)\left\|\left(S\left(t_{2}^{\eta} \theta\right)-S\left(t_{1}^{\eta} \theta\right)\right)\left(u_{0}+g_{n}\left(0, u_{0}\right)\right)\right\|_{\alpha-1} d \theta \\
& \leq \int_{0}^{\infty} \xi_{\eta}(\theta)\left[\int_{t_{1}}^{t_{2}} \frac{d}{d t} S\left(\left(t^{\eta} \theta\right)\right) d t\right]\left\|A^{\alpha-1}\left(u_{0}+g_{n}\left(0, u_{0}\right)\right)\right\| d \theta \\
& \leq \int_{0}^{\infty} \xi_{\eta}(\theta)\left[M_{1}\left(t_{2}-t_{1}\right)\right]\left\|A^{\alpha-1}\left(u_{0}+g_{n}\left(0, u_{0}\right)\right)\right\| d \theta \\
&(3.15) \quad \leq C_{1}\left(t_{2}-t_{1}\right),
\end{aligned}
$$

where $C_{1}=\left[\left\|u_{0}\right\|_{\alpha-1}+\left\|A^{\alpha-\beta-1}\right\|\left\|g_{n}\left(0, u_{0}\right)\right\|_{\beta}\right] M$.

For the second part of the right hand side of (3.14), we can see that

$$
\begin{aligned}
\left\|A^{\alpha-\beta-1}\right\| & \left\|A^{\beta} g_{n}\left(t_{2}, u\left(t_{2}\right)\right)-A^{\beta} g_{n}\left(t_{1}, u\left(t_{1}\right)\right)\right\| \\
& \leq\left\|A^{\alpha-\beta-1}\right\| L_{g}\left[\left(t_{2}-t_{1}\right)+\left\|u\left(t_{2}\right)-u\left(t_{1}\right)\right\|_{\alpha-1}\right] \\
& \leq C_{2}\left(t_{2}-t_{1}\right),
\end{aligned}
$$

where $C_{2}=\left\|A^{\alpha-\beta-1}\right\|\left[L_{g}(1+L)\right]$. To handle the third and fifth parts of the right hand side of (3.14), observe that

$$
\begin{aligned}
\int_{0}^{t_{1}} & \int_{0}^{\infty} \xi_{\eta}(\theta) \|\left[\eta \theta\left(t_{2}-s\right)^{\eta-1} A S\left(\left(t_{2}-s\right)^{\eta} \theta\right)-\eta \theta\left(t_{1}-s\right)^{\eta-1}\right. \\
& \left.A S\left(\left(t_{1}-s\right)^{\eta} \theta\right)\right] \| \\
& \times\left\|A^{\alpha-2} f_{n}(s, u(s), u[h(u(s), s)])\right\| d \theta d s \\
& \leq \int_{0}^{t_{1}} \int_{0}^{\infty} \xi_{\eta}(\theta)\left\|\left[\left.\frac{d}{d t} S\left((t-s)^{\eta} \theta\right)\right|_{t=t_{2}}-\left.\frac{d}{d t} S\left((t-s)^{\eta} \theta\right)\right|_{t=t_{1}}\right]\right\| \\
& \times\left\|A^{\alpha-2}\right\| N d \theta d s \\
& \leq \int_{0}^{t_{1}} \int_{0}^{\infty} \xi_{\eta}(\theta)\left[\int_{t_{1}}^{t_{2}}\left|\frac{d^{2}}{d t^{2}} S\left((t-s)^{\eta} \theta\right)\right| d t\right]\left\|A^{\alpha-2}\right\| N d \theta d s \\
& \leq \int_{0}^{t_{1}} \int_{0}^{\infty} \xi_{\eta}(\theta)\left[M_{2}\left(t_{2}-t_{1}\right)\right]\left\|A^{\alpha-2}\right\| N d \theta d s
\end{aligned}
$$

$$
\leq C_{3}\left(t_{2}-t_{1}\right)
$$


where $C_{3}=N M_{2}\left\|A^{\alpha-2}\right\| T_{0}$. Similarly, for the third part of (3.14), we have

$$
\begin{aligned}
\int_{0}^{t_{1}} & \int_{0}^{\infty} \xi_{\eta}(\theta) \|\left[\eta \theta\left(t_{2}-s\right)^{\eta-1} S\left(\left(t_{2}-s\right)^{\eta} \theta\right)\right. \\
& \left.-\eta \theta\left(t_{1}-s\right)^{\eta-1} S\left(\left(t_{1}-s\right)^{\eta} \theta\right)\right] A^{\alpha-\beta} \| \\
& \times\left\|A^{\beta} g_{n}(s, u(s))\right\| d \theta d s \\
& \leq C_{4}\left(t_{2}-t_{1}\right)
\end{aligned}
$$

where $C_{4}=N_{1} M_{2}\left\|A^{\alpha-\beta-1}\right\| T_{0}$. For the sixth part of (3.14), we have

$$
\begin{aligned}
\int_{t_{1}}^{t_{2}} & \int_{0}^{\infty} \xi_{\eta}(\theta)\left\|\eta \theta\left(t_{2}-s\right)^{\eta-1} A S\left(\left(t_{2}-s\right)^{\eta} \theta\right)\right\| \\
& \left\|A^{\alpha-2} f_{n}(s, u(s), u[h(u(s), s)])\right\| d \theta d s \\
& \leq\left.\int_{t_{1}}^{t_{2}} \int_{0}^{\infty} \xi_{\eta}(\theta)|| \frac{d}{d t} S\left((t-s)^{\eta} \theta\right)\right|_{t=t_{2}} \mid\left\|A^{\alpha-2}\right\| N d \theta d s \\
& \leq C_{5}\left(t_{2}-t_{1}\right),
\end{aligned}
$$

where $C_{5}=\left\|A^{\alpha-2}\right\| M_{1} N$. Finally, for the fourth part of (3.14), we have

$$
\begin{aligned}
\int_{t_{1}}^{t_{2}} & \int_{0}^{\infty} \xi_{\eta}(\theta)\left\|\eta \theta\left(t_{2}-s\right)^{\eta-1} A S\left(\left(t_{2}-s\right)^{\eta} \theta\right) A^{\alpha-\beta-1}\right\| \\
& \left\|A^{\beta} g_{n}(s, u(s))\right\| d \theta d s \\
& \leq C_{6}\left(t_{2}-t_{1}\right),
\end{aligned}
$$

where $C_{6}=\left\|A^{\alpha-\beta-1}\right\| M_{1} N_{1}$.

We use (3.15), (3.16) and (3.17)-(3.20) in (3.14) to get the following inequality:

$$
\left\|\left(\mathcal{F}_{n} u\right)\left(t_{2}\right)-\left(\mathcal{F}_{n} u\right)\left(t_{1}\right)\right\|_{\alpha-1} \leq L\left|t_{2}-t_{1}\right|
$$

where $L=\max \left\{C_{i}, i=1,2, \ldots 6\right\}$. Hence, $\mathcal{F}_{n}: \mathcal{C}_{T_{0}}^{\alpha-1} \rightarrow \mathcal{C}_{T_{0}}^{\alpha-1}$ follows.

Our next task is to show that $\mathcal{F}_{n}: \mathcal{W} \rightarrow \mathcal{W}$. Now, for $t \in\left[0, T_{0}\right]$ and $u \in \mathcal{W}$, we have

$$
\left\|\left(\mathcal{F}_{n} u\right)(t)-u_{0}\right\|_{\alpha}
$$




$$
\begin{aligned}
& \leq \int_{0}^{\infty} \theta \xi_{\eta}(\theta)\left\|\left(S\left(t^{\eta} \theta\right)-I\right) A^{\alpha}\left[u_{0}+g_{n}\left(0, u_{0}\right)\right]\right\| d \theta \\
&\left.+\left\|A^{\alpha-\beta}\right\| \| A^{\beta} g_{n}(s, u(s))\right)-A^{\beta} g_{n}(0, u(0)) \| \\
&+\eta \int_{0}^{t} \int_{0}^{\infty} \theta \xi_{\eta}(\theta)(t-s)^{\eta-1}\left\|S\left((t-s)^{\eta} \theta\right) A^{1+\alpha-\beta}\right\| \\
&\left.\left.\left.\| A^{\beta} g_{n}(s, u(s))\right)\right]\right) \| d \theta d s \\
&+\eta \int_{0}^{t} \int_{0}^{\infty} \theta \xi_{\eta}(\theta)(t-s)^{\eta-1}\left\|S\left((t-s)^{\eta} \theta\right) A^{\alpha}\right\| \\
&\left.\| f_{n}(s, u(s), u(h(u(s), s))]\right) \| d \theta d s \\
& \leq\left\|\left(S\left(t^{\eta} \theta\right)-I\right) A^{\alpha}\left[u_{0}+g_{n}\left(0, u_{0}\right)\right]\right\| \\
&+\left\|A^{\alpha-\beta}\right\| L_{g}\left[T_{0}+\left\|A^{-1}\right\| R\right] \\
&+C_{1+\alpha-\beta} N_{1} \frac{T_{0}^{\eta(\beta-\alpha)}}{\beta-\alpha}+C_{\alpha} N \frac{T_{0}^{\eta(1-\alpha)}}{1-\alpha} .
\end{aligned}
$$

Hence, from (3.7) and (3.8), we get

$$
\left\|\mathcal{F}_{n} u-u_{0}\right\|_{T_{0}, \alpha} \leq R
$$

Therefore, $\mathcal{F}_{n}: \mathcal{W} \rightarrow \mathcal{W}$.

Now, if $t \in\left[0, T_{0}\right]$ and $u, v \in \mathcal{W}$, then

$$
\begin{aligned}
& \left\|\left(\mathcal{F}_{n} u\right)(t)-\left(\mathcal{F}_{n} v\right)(t)\right\|_{\alpha} \\
& \leq\left\|A^{\alpha-\beta}\right\|\left\|A^{\beta} g_{n}(t, u(s))-A^{\beta} g_{n}(t, v(s))\right\| \\
& \quad+\eta \int_{0}^{t} \int_{0}^{\infty} \theta \xi_{\eta}(\theta)(t-s)^{\eta-1}\left\|S\left((t-s)^{\eta} \theta\right) A^{1+\alpha-\beta}\right\| \\
& \left.\times \| A^{\beta} g_{n}(s, u(s))\right)-A^{\beta} g_{n}(s, v(s)) \| d \theta d s \\
& \quad+\eta \int_{0}^{t} \int_{0}^{\infty} \theta \xi_{\eta}(\theta)(t-s)^{\eta-1}\left\|S\left((t-s)^{\eta} \theta\right) A^{\alpha}\right\| \\
& \| f_{n}(s, u(s), u(h[u(s), s])) \\
& \left.\quad-f_{n}(s, v(s), v(h[v(s), s]))\right) \| d \theta d s .
\end{aligned}
$$

We have the following inequalities:

$$
\left.\| A^{\beta} g_{n}(s, u(s))\right)-A^{\beta} g_{n}(t, v(t))\left\|\leq L_{g}\right\| A^{-1}\|\| u-v \|_{T_{0}, \alpha},
$$




$$
\begin{aligned}
\left\|f_{n}(s, u(s), u[h(u(s), s)])-f_{n}(s, v(s), v[h(v(s), s)])\right\| \\
\leq L_{f}\left(2+L L_{h}\right)\|u-v\|_{T_{0}, \alpha} .
\end{aligned}
$$

We use the inequalities (3.23) and (3.24) in (3.22) and get

$$
\begin{aligned}
\left\|\left(\mathcal{F}_{n} u\right)(t)-\left(\mathcal{F}_{n} v\right)(t)\right\|_{\alpha} \leq & {\left[L_{g}\left\|A^{\alpha-\beta-1}\right\|+C_{1+\alpha-\beta} L_{g}\left\|A^{-1}\right\| \frac{T_{0}^{\eta(\beta-\alpha)}}{\beta-\alpha}\right.} \\
& \left.+C_{\alpha} L_{f}\left(2+L L_{h}\right) \frac{T_{0}^{\eta(1-\alpha)}}{1-\alpha}\right]\|u-v\|_{T_{0}, \alpha} .
\end{aligned}
$$

Hence, from inequality (3.4), we get the following inequality:

$$
\left\|\mathcal{F}_{n} u-\mathcal{F}_{n} v\right\|_{T_{0}, \alpha}<\|u-v\|_{T_{0}, \alpha},
$$

i.e., the map $\mathcal{F}_{n}$ is a contraction on $\mathcal{W}$. Therefore, the map $\mathcal{F}_{n}$ has a unique fixed point $u_{n} \in \mathcal{W}$, given by

$$
\begin{aligned}
u_{n}(t)= & \int_{0}^{\infty} \theta \xi_{\eta}(\theta) S(t)\left[u_{0}+g_{n}\left(0, u_{0}\right)\right] d \theta-g_{n}\left(t, u_{n}(t)\right) \\
& \left.+\eta \int_{0}^{t} \int_{0}^{\infty} \theta \xi_{\eta}(\theta)(t-s)^{\eta-1} A S\left((t-s)^{\eta} \theta\right) g_{n}\left(s, u_{n}(s)\right)\right) d \theta d s \\
& +\eta \int_{0}^{t} \int_{0}^{\infty} \theta \xi_{\eta}(\theta)(t-s)^{\eta-1} S\left((t-s)^{\eta} \theta\right)
\end{aligned}
$$

$$
f_{n}\left(s, u_{n}(s), u_{n}\left(h\left(u_{n}(s), s\right)\right)\right) d \theta d s, \quad t \in\left(0, T_{0}\right] .
$$

This completes the proof of Theorem 3.1.

Lemma 3.2. Assume that assumptions $(\mathrm{H} 1)-(\mathrm{H} 3)$ are satisfied. We have the following results

(i) If $u_{0} \in D\left(A^{\alpha}\right)$, then $u_{n}(t) \in D\left(A^{\vartheta}\right)$ for all $t \in\left(0, T_{0}\right]$,

(ii) If $u_{0} \in D(A)$, then $u_{n}(t) \in D\left(A^{\vartheta}\right)$ for all $t \in\left(0, T_{0}\right]$,

for $0<\vartheta<\beta<1$.

Proof. Since we have proved that $u_{n} \in \mathcal{W} \subseteq \mathcal{C}_{T_{0}}^{\alpha-1}$, then $u_{n}$ must be Hölder continuous on $\left[0, T_{0}\right]$. Furthermore, the inequalities $(\mathrm{H} 2)-(\mathrm{H} 4)$ imply the Hölder continuity of $f\left(t, u_{n}(t), u_{n}\left(h\left(u_{n}(t), t\right)\right)\right)$ and $g\left(t, u_{n}(t)\right)$ 
on $\left[0, T_{0}\right]$. We also note that $[\mathbf{2 6}$, Theorem 3.2 , page 111$]$

$$
\eta \int_{0}^{t} \int_{0}^{\infty} \theta \xi_{\eta}(\theta)(t-s)^{\eta-1} A S\left((t-s)^{\eta} \theta\right) g_{n}\left(s, u_{n}(s)\right) d \theta d s \in D(A) .
$$

Hence, we can easily prove that $u_{n}(t) \in D(A)$. For more details, we refer to [5, Theorem 2.2]. Part (i) follows from (ii) and the fact that $D(A) \subset D\left(A^{\vartheta}\right), 0<\vartheta \leq 1$ (see Lemma 2.1 (ii)).

Lemma 3.3. Suppose that assumptions $(\mathrm{H} 1)-(\mathrm{H} 4)$ are satisfied. We have the following inequalities:

(i) If $u_{0} \in D\left(A^{\alpha}\right)$, then for any $t_{0} \in\left(0, T_{0}\right]$

$$
\left\|u_{n}(t)\right\|_{\vartheta} \leq U_{t_{0}}, \quad t \in\left[t_{0}, T_{0}\right], n=1,2, \ldots,
$$

for some constant $U_{t_{0}}$, independent of $n$.

(ii) If $u_{0} \in D(A)$, then there exists a constant $U_{0}$ such that

$$
\left\|u_{n}(t)\right\|_{\vartheta} \leq U_{0}, \quad t \in\left[0, T_{0}\right], n=1,2, \ldots .
$$

Proof. Let $u_{0} \in D\left(A^{\alpha}\right)$. Applying $A^{\vartheta}$ on both sides of (3.26), for $t \in\left[t_{0}, T_{0}\right]$ and $\alpha<\vartheta<\beta$, we have

$$
\begin{aligned}
\left\|u_{n}(t)\right\|_{\vartheta} \leq & \int_{0}^{\infty} \xi_{\eta}(\theta) \| A^{\vartheta} S\left(t^{\eta} \theta\right)\left(u_{0}+g_{n}\left(0, u_{0}\right) \| d \theta\right. \\
& +\left\|A^{\vartheta-\beta}\right\|\left\|A^{\beta} g_{n}\left(t, u_{n}(t)\right)\right\| \\
& +\eta \int_{0}^{t} \int_{0}^{\infty} \theta \xi_{\eta}(\theta)(t-s)^{\eta-1}\left\|A^{1+\vartheta-\beta} S\left((t-s)^{\eta} \theta\right)\right\| \\
\times & \left\|A^{\beta} g_{n}\left(s, u_{n}(s)\right)\right\| d \theta d s \\
& +\eta \int_{0}^{t} \int_{0}^{\infty} \theta \xi_{\eta}(\theta)(t-s)^{\eta-1} \\
\times & \left.\| S\left((t-s)^{\eta} \theta\right)\right) A^{\vartheta} \| \\
& \left\|f_{n}\left(s, u_{n}(s), u_{n}\left(h\left(u_{n}(s), s\right)\right)\right)\right\| d \theta d s \\
\leq & C_{\vartheta} t_{0}^{-\eta \vartheta}\left(\left\|u_{0}\right\|+\left\|g_{n}\left(0, u_{0}\right)\right\|\right. \\
& +\left\|A^{\vartheta-\beta}\right\| N_{1} \\
& +C_{1+\vartheta-\beta} N_{1} \frac{T^{\eta(\beta-\vartheta)}}{\beta-\vartheta}+C_{\vartheta} N \frac{T^{\eta(1-\vartheta)}}{1-\vartheta} \leq U_{t_{0}} .
\end{aligned}
$$


Similarly, we can find the estimate

$$
\begin{aligned}
\left\|u_{n}(t)\right\|_{\vartheta} \leq & M\left(\left\|A^{\vartheta} u_{0}\right\|+\left\|g_{n}\left(0, \widetilde{u_{0}} \|_{\vartheta}\right)+\right\| A^{\vartheta-\beta} \| N_{1}\right. \\
& +C_{1+\vartheta-\beta} N_{1} \frac{T^{\eta(\beta-\vartheta)}}{\beta-\vartheta}+C_{\vartheta} N \frac{T^{\eta(1-\vartheta)}}{1-\vartheta} \leq U_{0},
\end{aligned}
$$

for given $u_{0} \in D(A)$ and $t \in\left(0, T_{0}\right]$.

4. Convergence of solutions. In this section we establish the convergence of the solution $u_{n} \in H_{\alpha}\left(T_{0}\right)$ of each approximate integral equation to a unique solution $u$ of (3.9).

Theorem 4.1. Let us assume that conditions (H1)-(H3) are satisfied. If $u_{0} \in D\left(A^{\alpha}\right)$, then for $t_{0} \in\left(0, T_{0}\right]$

$$
\left\|u_{n}-u_{m}\right\|_{T_{0}, \alpha} \longrightarrow 0, \quad \text { as } m, n \rightarrow \infty,
$$

i.e., $u_{n}$ is a Cauchy sequence in $\mathcal{W}$ on $\left[t_{0}, T_{0}\right]$.

Proof. Let $0<\alpha<\vartheta<\beta$. For $n \geq m$, we have

$$
\begin{aligned}
& \left\|f_{n}\left(t, u_{n}(t), u_{n}\left[h\left(u_{n}(t), t\right)\right]\right)-f_{m}\left(t, u_{m}(t), u_{m}\left[h\left(u_{m}(t), t\right)\right]\right)\right\| \\
& \leq \| f_{n}\left(t, u_{n}(t), u_{n}\left[h\left(u_{n}(t), t\right)\right]\right) \\
& \quad-f_{n}\left(t, u_{m}(t), u_{m}\left[h\left(u_{m}(t), t\right)\right]\right) \| \\
& \quad+\| f_{n}\left(t, u_{m}(t), u_{m}\left[h\left(u_{m}(t), t\right)\right]\right) \\
& \quad-f_{m}\left(t, u_{m}(t), u_{m}\left[h\left(u_{m}(t), t\right)\right]\right) \| \\
& \leq L_{f}\left(2+L L_{h}\right)\left\|u_{n}(t)-u_{m}(t)\right\|_{\alpha} \\
& \quad+L_{f}\left[\left\|\left(P^{n}-P^{m}\right) u_{m}(t)\right\|_{\alpha}\right. \\
& \left.\quad+\left\|A^{-1}\right\|\left\|\left(P^{n}-P^{m}\right) u_{m}\left(h\left(u_{m}(t), t\right)\right)\right\|_{\alpha}\right] .
\end{aligned}
$$

Also,

$$
\begin{aligned}
\left\|\left(P^{n}-P^{m}\right) u_{m}(t)\right\|_{\alpha} & \leq\left\|A^{\alpha-\vartheta}\left(P^{n}-P^{m}\right) A^{\vartheta} u_{m}(t)\right\| \\
& \leq \frac{1}{\lambda_{m}^{\vartheta-\alpha}}\left\|A^{\vartheta} u_{m}(t)\right\| .
\end{aligned}
$$

Thus, we have

$$
\left\|f_{n}\left(t, u_{n}(t), u_{n}\left[h\left(u_{n}(t), t\right)\right]\right)-f_{m}\left(t, u_{m}(t), u_{m}\left[h\left(u_{m}(t), t\right)\right]\right)\right\|
$$




$$
\begin{aligned}
\leq L_{f}\left(2+L L_{h}\right) \| u_{n}(t)-u_{m}(t) & \|_{\alpha}+L_{f}\left[\frac{1}{\lambda_{m}^{\vartheta-\alpha}}\left\|A^{\vartheta} u_{m}(t)\right\|\right. \\
+ & \left.\frac{\left\|A^{-1}\right\|}{\lambda_{m}^{\vartheta-\alpha}}\left\|A^{\vartheta} u_{m}\left(h\left(u_{m}(t), t\right)\right)\right\|\right]
\end{aligned}
$$

Similarly,

$$
\begin{aligned}
\| A^{\beta} g_{n}( & \left.t, u_{n}(t)\right)-A^{\beta} g_{m}\left(t, u_{m}(t)\right) \| \\
\leq & \left\|A^{\beta} g_{n}\left(t, u_{n}(t)\right)-A^{\beta} g_{n}\left(t, u_{m}(t)\right)\right\| \\
& \quad+\left\|A^{\beta} g_{n}\left(t, u_{m}(t)\right)-A^{\beta} g_{m}\left(t, u_{m}(t)\right)\right\| \\
\leq & L_{g}\left\|A^{-1}\right\|\left[\left\|u_{n}(t)-u_{m}(t)\right\|_{\alpha}+\frac{1}{\lambda_{m}^{\vartheta-\alpha}}\left\|A^{\vartheta} u_{m}(t)\right\|\right] .
\end{aligned}
$$

Now, for $0<t_{0}^{\prime}<t_{0}$, we may write

$$
\begin{aligned}
\left\|u_{n}(t)-u_{m}(t)\right\|_{\alpha} \leq & \int_{0}^{\infty} \xi_{\eta}(\theta)\left\|S\left(t^{\eta} \theta\right) A^{\alpha}\left(g_{n}\left(0, u_{0}\right)-g_{m}\left(0, u_{0}\right)\right)\right\| d \theta \\
& +\left\|A^{\alpha-\beta}\right\|\left\|A^{\beta} g_{n}\left(t, u_{n}(t)\right)-A^{\beta} g_{m}\left(t, u_{m}(t)\right)\right\| \\
& +\eta\left(\int_{0}^{t_{0}^{\prime}}+\int_{t_{0}^{\prime}}^{t}\right) \int_{0}^{\infty} \theta \xi_{\eta}(\theta)(t-s)^{\eta-1} \\
& \left.\| A^{1+\alpha-\beta} S\left((t-s)^{\eta} \theta\right)\right) \| \\
\times & \left\|A^{\beta} g_{n}\left(s, u_{n}(s)\right)-A^{\beta} g_{m}\left(s, u_{m}(s)\right)\right\| d \theta d s \\
& +\eta\left(\int_{0}^{t_{0}^{\prime}}+\int_{t_{0}^{\prime}}^{t}\right) \int_{0}^{\infty} \theta \xi_{\eta}(\theta)(t-s)^{\eta-1} \\
& \left\|A^{\alpha} S\left((t-s)^{\eta} \theta\right)\right\| \\
\times & \| f_{n}\left(s, u_{n}(s), u_{n}\left(h\left(u_{n}(s), s\right)\right)\right) \\
& -f_{m}\left(s, u_{m}(s), u_{m}\left(h\left(u_{m}(s), s\right)\right)\right) \| d \theta d s .
\end{aligned}
$$

We estimate the first term as

$$
\begin{aligned}
\int_{0}^{\infty} & \xi_{\eta}(\theta)\left\|S\left(t^{\eta} \theta\right) A^{\alpha}\left(g_{n}\left(0, u_{0}\right)-g_{m}\left(0, u_{0}\right)\right)\right\| d \theta \\
& \leq \int_{0}^{\infty} \xi_{\eta}(\theta) M\left\|A^{\alpha-\beta}\right\|\left\|A^{\beta} g\left(0, P^{n} u_{0}\right)-A^{\beta} g\left(0, P^{m} u_{0}\right)\right\| d \theta \\
& \leq M\left\|A^{\alpha-\beta-1}\right\| L_{g}\left\|\left(P^{n}-P^{m}\right) A^{\alpha} u_{0}\right\| \int_{0}^{\infty} \xi_{\eta}(\theta) d \theta
\end{aligned}
$$




$$
\begin{aligned}
& \leq M\left\|A^{\alpha-\beta-1}\right\| L_{g}\left\|\left(P^{n}-P^{m}\right) A^{\alpha} u_{0}\right\| \\
& \leq \frac{1}{\lambda_{m}^{\vartheta-\alpha}} M\left\|A^{\alpha-\beta-1}\right\| L_{g}\|\| A^{\vartheta} u_{0} \| .
\end{aligned}
$$

The first and third integrals are estimated as

$$
\begin{aligned}
& \eta \int_{0}^{t_{0}^{\prime}} \int_{0}^{\infty} \theta \xi_{\eta}(\theta)(t-s)^{\eta-1}\left\|A^{1+\alpha-\beta} S\left((t-s)^{\eta} \theta\right)\right\| \\
&\left\|A^{\beta} g_{n}\left(s, u_{n}(s)\right)-A^{\beta} g_{m}\left(s, u_{m}(s)\right)\right\| d \theta d s \\
& \leq \frac{2 C_{1+\alpha-\beta} N_{1}}{\beta-\alpha}\left(t^{\eta(\beta-\alpha)}-\left(t-t_{0}^{\prime}\right)^{\eta(\beta-\alpha)}\right) \\
& \leq \frac{2 C_{1+\alpha-\beta} N_{1}}{\beta-\alpha}\left(t-\delta_{1} t_{0}^{\prime}\right)^{\eta(\beta-\alpha)-1} t_{0}^{\prime}, \quad 0<\delta_{1}<1 \\
& \leq \frac{2 C_{1+\alpha-\beta} N_{1}}{\beta-\alpha}\left(t_{0}-t_{0}^{\prime}\right)^{\eta(\beta-\alpha)-1} t_{0}^{\prime} . \\
& \eta \int_{0}^{t_{0}^{\prime}} \int_{0}^{\infty} \xi_{\eta}(\theta)(t-s)^{\eta-1}\left\|A^{\alpha} S\left((t-s)^{\eta} \theta\right)\right\| \\
& \| f_{n}\left(s, u_{n}(s), u_{n}\left(h\left(u_{n}(s), s\right)\right)\right) \\
&-f_{m}\left(s, u_{m}(s), u_{m}\left(h\left(u_{m}(s), s\right)\right)\right) \| d \theta d s \\
& \leq \frac{2 C_{\alpha} N}{1-\alpha}\left(t^{\eta(1-\alpha)}-\left(t-t_{0}^{\prime}\right)^{\eta(1-\alpha)}\right) \\
& \leq \frac{2 C_{\alpha} N}{1-\alpha}\left(t-\delta_{2} t_{0}^{\prime}\right)^{\eta(1-\alpha)-1} t_{0}^{\prime}, \quad 0<\delta_{2}<1 \\
& \leq \frac{2 C_{\alpha} N}{1-\alpha}\left(t_{0}-t_{0}^{\prime}\right)^{\eta(1-\alpha)-1} t_{0}^{\prime} .
\end{aligned}
$$

For the second and fourth integrals, we have

$$
\begin{gathered}
\eta \int_{t_{0}^{\prime}}^{t} \int_{0}^{\infty} \theta \xi_{\eta}(\theta)(t-s)^{\eta-1}\left\|A^{1+\alpha-\beta} S\left((t-s)^{\eta} \theta\right)\right\| \\
\left\|A^{\beta} g_{n}\left(s, u_{n}(s)\right)-A^{\beta} g_{m}\left(s, u_{m}(s)\right)\right\| d \theta d s \\
\leq \eta C_{1+\alpha-\beta} L_{g}\left\|A^{-1}\right\|\left(\frac{U_{t_{0}^{\prime}} T_{0}^{\eta(\beta-\alpha)}}{\lambda_{m}^{\vartheta-\alpha} \eta(\beta-\alpha)}\right. \\
\left.+\int_{t_{0}^{\prime}}^{t}(t-s)^{\eta(\beta-\alpha)-1}\left\|u_{n}(s)-u_{m}(s)\right\|_{\alpha} d s\right)
\end{gathered}
$$




$$
\begin{aligned}
\eta \int_{t_{0}^{\prime}}^{t} & \int_{0}^{\infty} \theta \xi_{\eta}(\theta)(t-s)^{\eta-1}\left\|A^{\alpha} S\left((t-s)^{\eta} \theta\right)\right\| \\
& \times f_{n}\left(s, u_{n}(s), u_{n}\left[h\left(u_{n}(s), s\right)\right]\right) \\
& \quad-f_{m}\left(s, u_{m}(s), u_{m}\left[h\left(u_{m}(s), s\right)\right]\right) d \theta d s \\
& \leq \eta C_{\alpha} L_{f}\left(\left(1+\left\|A^{-1}\right\|\right) \frac{U_{t_{0}^{\prime}} T_{0}^{\eta(1-\alpha)}}{\lambda_{m}^{\vartheta-\alpha} \eta(1-\alpha)}\right. \\
& \left.+\left(2+L L_{h}\right) \int_{t_{0}^{\prime}}^{t}(t-s)^{\eta(1-\alpha)-1}\left\|u_{n}(s)-u_{m}(s)\right\|_{\alpha} d s\right)
\end{aligned}
$$

Therefore,

$$
\begin{aligned}
\left\|u_{n}(t)-u_{m}(t)\right\|_{\alpha} \leq & \frac{1}{\lambda_{m}^{\vartheta-\alpha}} M\left\|A^{\alpha-\beta-1}\right\| L_{g}\|\| A^{\vartheta} u_{0} \| \\
& +\left\|A^{\alpha-\beta-1}\right\| L_{g}\left(\left\|u_{n}(t)-u_{m}(t)\right\|_{\alpha}+\frac{U_{t_{0}^{\prime}}}{\lambda_{m}^{\vartheta-\alpha}}\right) \\
& +2\left(\frac{C_{1+\alpha-\beta} N_{1}}{\left(t_{0}-t_{0}^{\prime}\right)^{1-\eta(\beta-\alpha)}}+\frac{C_{\alpha} N}{\left(t_{0}-t_{0}^{\prime}\right)^{1-\eta(1-\alpha)}}\right) t_{0}^{\prime} \\
& +C_{\alpha, \beta} \frac{U_{t_{0}^{\prime}}}{\lambda_{m}^{\vartheta-\alpha}} \\
& +\int_{t_{0}^{\prime}}^{t}\left(\frac{C_{\alpha} L_{f}\left(2+L L_{h}\right)}{(t-s)^{\eta(\alpha-1)+1}}+\frac{C_{1+\alpha-\beta} L_{g}\left\|A^{-1}\right\|}{(t-s)^{\eta(\alpha-\beta)+1}}\right) \\
& \left\|u_{n}(s)-u_{m}(s)\right\|_{\alpha} d s,
\end{aligned}
$$

where

$$
C_{\alpha, \beta}=\left(1+\left\|A^{-1}\right\|\right) C_{\alpha} L_{f} \frac{T_{0}^{\eta(1-\alpha)}}{1-\alpha}+C_{1+\alpha-\beta} L_{g}\left\|A^{-1}\right\| \frac{T_{0}^{\eta(\beta-\alpha)}}{\beta-\alpha} .
$$

Since $\left\|A^{\alpha-\beta-1}\right\| L_{g}<1$, we have

$$
\begin{aligned}
\left\|u_{n}(t)-u_{m}(t)\right\|_{\alpha} \leq & \frac{1}{\left(1-\left\|A^{\alpha-\beta-1}\right\| L_{g}\right)} \\
& \times\left\{\left\|A^{\alpha-\beta-1}\right\| L_{g}\left(M \frac{1}{\lambda_{m}^{\vartheta-\alpha}}\left\|A^{\vartheta} u_{0}\right\|+\frac{U_{t_{0}^{\prime}}}{\lambda_{m}^{\vartheta-\alpha}}\right)\right. \\
& +2\left(\frac{C_{1+\alpha-\beta} N_{1}}{\left(t_{0}-t_{0}^{\prime}\right)^{1-\eta(\beta-\alpha)}}\right.
\end{aligned}
$$




$$
\begin{aligned}
& \left.\left.+\frac{C_{\alpha} N}{\left(t_{0}-t_{0}^{\prime}\right)^{1-\eta(1-\alpha)}}\right) t_{0}^{\prime}+C_{\alpha, \beta} \frac{U_{t_{0}^{\prime}}}{\lambda_{m}^{\vartheta-\alpha}}\right\} \\
& +\int_{t_{0}^{\prime}}^{t}\left(\frac{C_{\alpha} L_{f}\left(2+L L_{h}\right)}{(t-s)^{\eta(\alpha-1)+1}}\right. \\
& \left.+C_{1+\alpha-\beta} L_{g}\left\|A^{-1}\right\|(t-s)^{\eta(\alpha-\beta)+1}\right) \\
& \left\|u_{n}(s)-u_{m}(s)\right\|_{\alpha} d s .
\end{aligned}
$$

Applying Gronwall's inequality and estimating $t-s$ by $T_{0}$, we get the following:

$$
\begin{aligned}
\left\|u_{n}(t)-u_{m}(t)\right\|_{\alpha} \leq & \frac{1}{\left(1-\left\|A^{\alpha-\beta-1}\right\| L_{g}\right)} \\
& \left\{\left(\left\|A^{\alpha-\beta-1}\right\| L_{g} M\left\|A^{\vartheta} u_{0}\right\|\right.\right. \\
& \left.+\left\|A^{\alpha-\beta-1}\right\| L_{g} U_{t_{0}^{\prime}}+C_{\alpha, \beta} U_{t_{0}^{\prime}}\right) \frac{1}{\lambda_{m}^{\vartheta-\alpha}} \\
& \left.+2\left(\frac{C_{1+\alpha-\beta} N_{1}}{\left(t_{0}-t_{0}^{\prime}\right)^{1-\eta(\beta-\alpha)}}+\frac{C_{\alpha} N}{\left(t_{0}-t_{0}^{\prime}\right)^{1-\eta(1-\alpha)}}\right) t_{0}^{\prime}\right\} C .
\end{aligned}
$$

Letting $m \rightarrow \infty$ and taking the supremum over $\left[t_{0}, T_{0}\right]$, we obtain

$$
\begin{aligned}
& \left\|u_{n}-u_{m}\right\|_{T_{0}, \alpha} \\
& \leq \frac{2}{\left(1-\left\|A^{\alpha-\beta-1}\right\| L_{g}\right)}\left(\frac{C_{1+\alpha-\beta} N_{1}}{\left(t_{0}-t_{0}^{\prime}\right)^{1-\eta(\beta-\alpha)}}+\frac{C_{\alpha} N}{\left(t_{0}-t_{0}^{\prime}\right)^{1-\eta(1-\alpha)}}\right) t_{0}^{\prime} C .
\end{aligned}
$$

As $t_{0}^{\prime}$ is arbitrary, the right hand side may be made as small as desired by taking $t_{0}^{\prime}$ sufficiently small. This completes the proof of Theorem 4.1 .

Similarly, we can prove the following corollary.

Corollary 4.2. If $u_{0} \in D(A)$, then

$$
\left\|u_{n}-u_{m}\right\|_{T_{0}, \alpha} \longrightarrow 0, \quad \text { as } m, n \rightarrow \infty,
$$

i.e., $u_{n}$ is a Cauchy sequence in $\mathcal{W}$ on $\left(0, T_{0}\right]$. 
With the help of Theorems 3.1 and 4.1, we have the following result for the convergence of solutions to each of the approximate integral equations.

Theorem 4.3. Let us suppose that assumptions (H1)-(H4) are satisfied, and let $u_{0} \in D\left(A^{\alpha}\right)$ or $D(A)$. Then there exists a unique function $u_{n} \in \mathcal{W}$,

$$
\begin{aligned}
u_{n}(t)= & \int_{0}^{\infty} \xi_{\eta}(\theta) S\left(t^{\eta} \theta\right)\left[u(0)+g_{n}\left(0, u_{0}\right)\right] d \theta-g_{n}\left(t, u_{n}(t)\right) \\
& +\eta \int_{0}^{t} \int_{0}^{\infty} \theta \xi_{\eta}(\theta)(t-s)^{\eta-1} A S\left((t-s)^{\eta} \theta\right) g_{n}\left(s, u_{n}(s)\right) d \theta d s \\
& +\eta \int_{0}^{t} \int_{0}^{\infty} \theta \xi_{\eta}(\theta)(t-s)^{\eta-1} S\left((t-s)^{\eta} \theta\right) \\
& \times f_{n}\left(s, u_{n}(s), u_{n}\left(h_{n}\left(u_{n}(s), s\right)\right)\right) d \theta d s, \quad t \in\left(0, T_{0}\right]
\end{aligned}
$$

and $u \in \mathcal{W}$,

$$
\begin{aligned}
u(t)= & \int_{0}^{\infty} \xi_{\eta}(\theta) S\left(t^{\eta} \theta\right)\left[u(0)+g\left(0, u_{0}\right)\right] d \theta-g(t, u(t)) \\
& +\eta \int_{0}^{t} \int_{0}^{\infty} \theta \xi_{\eta}(\theta)(t-s)^{\eta-1} A S\left((t-s)^{\eta} \theta\right) g(s, u(s)) d \theta d s \\
& +\eta \int_{0}^{t} \int_{0}^{\infty} \theta \xi_{\eta}(\theta)(t-s)^{\eta-1} S\left((t-s)^{\eta} \theta\right) \\
\times & f(s, u(s), u(h(u(s), s))) d \theta d s, \quad t \in\left(0, T_{0}\right],
\end{aligned}
$$

such that $u_{n} \rightarrow u$ as $n \rightarrow \infty$ in $\mathcal{W}$ and $u$ satisfies $(3.9)$ on $\left(0, T_{0}\right]$.

5. Faedo-Galerkin approximations. In this section, we will study the Faedo-Galerkin approximation solution of (1.1) and prove the convergence result for such an approximation.

We have proved a unique solution $u \in \mathcal{W}$ of the integral equation:

$$
\begin{aligned}
u(t)= & \int_{0}^{\infty} \xi_{\eta}(\theta) S\left(t^{\eta} \theta\right)\left[u(0)+g\left(0, u_{0}\right)\right] d \theta-g_{n}(t, u(t)) \\
& +\eta \int_{0}^{t} \int_{0}^{\infty} \theta \xi_{\eta}(\theta)(t-s)^{\eta-1} A S\left((t-s)^{\eta} \theta\right) g(s, u(s)) d \theta d s
\end{aligned}
$$




$$
+\eta \int_{0}^{t} \int_{0}^{\infty} \theta \xi_{\eta}(\theta)(t-s)^{\eta-1} S\left((t-s)^{\eta} \theta\right)
$$

$$
f(s, u(s), u(h(u(s), s))) d \theta d s, \quad t \in\left[0, T_{0}\right] .
$$

Then it has the representation

$$
u(t)=\sum_{i=0}^{\infty} \alpha_{i}(t) \phi_{i}, \quad \alpha_{i}(t)=\left(u(t), \phi_{i}\right), \quad i=0,1, \ldots ;
$$

where the $\phi_{i}$ 's are defined in (H1).

Also, we have a unique solution $u_{n} \in \mathcal{W}$ of the approximate integral equation

$$
\begin{aligned}
u_{n}(t)= & \int_{0}^{\infty} \xi_{\eta}(\theta) S\left(t^{\eta} \theta\right)\left[u(0)+g_{n}\left(0, u_{0}\right)\right] d \theta-g_{n}\left(t, u_{n}(t)\right) \\
& +\eta \int_{0}^{t} \int_{0}^{\infty} \theta \xi_{\eta}(\theta)(t-s)^{\eta-1} A S\left((t-s)^{\eta} \theta\right) g_{n}\left(s, u_{n}(s)\right) d \theta d s \\
& +\eta \int_{0}^{t} \int_{0}^{\infty} \theta \xi_{\eta}(\theta)(t-s)^{\eta-1} S\left((t-s)^{\eta} \theta\right)
\end{aligned}
$$

(5.3) $f_{n}\left(s, u_{n}(s), u_{n}\left(h\left(u_{n}(s), s\right)\right)\right) d \theta d s, \quad t \in\left[0, T_{0}\right]$.

Let $P^{n} u_{n}(t)=\widehat{u}_{n}(t)$ be the orthogonal projection of (5.3) on the first $n$ elements of $\left\{\phi_{i}\right\}$ satisfying the following equation:

$$
\begin{aligned}
\widehat{u}_{n}(t)= & \int_{0}^{\infty} \xi_{\eta}(\theta) S\left(t^{\eta} \theta\right) P^{n}\left[u(0)+g_{n}\left(0, u_{0}\right)\right] d \theta-P^{n} g_{n}\left(t, u_{n}(t)\right) \\
& +\eta \int_{0}^{t} \int_{0}^{\infty} \theta \xi_{\eta}(\theta)(t-s)^{\eta-1} A S\left((t-s)^{\eta} \theta\right) P^{n} g_{n}\left(s, u_{n}(s)\right) d \theta d s \\
& +\eta \int_{0}^{t} \int_{0}^{\infty} \theta \xi_{\eta}(\theta)(t-s)^{\eta-1} S\left((t-s)^{\eta} \theta\right) P^{n}
\end{aligned}
$$

$$
f_{n}\left(s, u_{n}(s), u_{n}\left(h\left(u_{n}(s), s\right)\right)\right) d \theta d s, \quad t \in\left[0, T_{0}\right] .
$$

Using (3.10) and (3.11) in (5.4), we get

$$
\widehat{u}_{n}(t)=\int_{0}^{\infty} \xi_{\eta}(\theta) S\left(t^{\eta} \theta\right) P^{n}\left[u(0)+g_{n}\left(0, u_{0}\right)\right] d \theta-P^{n} g\left(t, \widehat{u}_{n}(t)\right)
$$




$$
\begin{aligned}
& +\eta \int_{0}^{t} \int_{0}^{\infty} \theta \xi_{\eta}(\theta)(t-s)^{\eta-1} A S\left((t-s)^{\eta} \theta\right) P^{n} g\left(s, \widehat{u}_{n}(s)\right) d \theta d s \\
& +\eta \int_{0}^{t} \int_{0}^{\infty} \theta \xi_{\eta}(\theta)(t-s)^{\eta-1} S\left((t-s)^{\eta} \theta\right) P^{n}
\end{aligned}
$$

$$
f\left(s, \widehat{u}_{n}(s), \hat{u}_{n}\left(h\left(\widehat{u}_{n}(s), s\right)\right)\right) d \theta d s, \quad t \in\left[0, T_{0}\right] .
$$

The solution $\widehat{u}_{n}$ of (5.5) has the following representation

$$
\widehat{u}_{n}(t)=\sum_{i=0}^{n} \alpha_{i}^{n}(t) \phi_{i}, \quad \alpha_{i}^{n}(t)=\left(\hat{u}_{n}(t), \phi_{i}\right), \quad i=0,1, \ldots ;
$$

Then we get a system of equations from (5.4) and (5.6)

$$
\begin{aligned}
\frac{d^{\beta}}{d t^{\beta}} & {\left[\alpha_{i}^{n}(t)+H_{i}^{n}\left(t, \alpha_{0}^{n}, \alpha_{1}^{n}, \cdots, \alpha_{n}^{n}\right)\right]+\lambda_{i} \alpha_{i}^{n}(t) } \\
& =F_{i}^{n}\left(t, \alpha_{0}^{n}, \alpha_{1}^{n}, \cdots, \alpha_{n}^{n}, \tau_{0}^{n}, \tau_{1}^{n}, \cdots, \tau_{n}^{n}\right) \\
\alpha_{i}^{n}(0) & =u_{i},
\end{aligned}
$$

where

$$
\begin{aligned}
F_{i}^{n} & =\left(f\left(t, \sum_{i=0}^{n} \alpha_{i}^{n} \phi_{i}, \sum_{i=0}^{n} \tau_{i}^{n} \phi_{i}\right), \phi_{i}\right), \\
H_{i}^{n} & =\left(g\left(t, \sum_{i=0}^{n} \alpha_{i}^{n} \phi_{i}\right), \phi_{i}\right), \\
\tau_{i}^{n} & =\alpha_{i}^{n}\left(h\left(\alpha_{0}^{n}, \alpha_{1}^{n}, \ldots, \alpha_{n}^{n}, t\right)\right)
\end{aligned}
$$

and $u_{i}=\left(u_{0}, \phi_{i}\right)$ for $i=1,2, \ldots, n$. Convergence of $\alpha_{i}^{n}(t) \rightarrow \alpha_{i}(t)$ follows from the following theorem and the fact that

$$
\begin{aligned}
A^{\alpha}\left[u(t)-\widehat{u}_{n}(t)\right] & =A^{\alpha}\left[\sum_{i=0}^{\infty}\left(\alpha_{i}(t)-\alpha_{i}^{n}(t)\right) \phi_{i}\right] \\
& =\sum_{i=0}^{\infty} \lambda_{i}^{\alpha}\left(\alpha_{i}(t)-\alpha_{i}^{n}(t)\right) \phi_{i} .
\end{aligned}
$$


Thus, we have

$$
\left\|A^{\alpha}\left[u(t)-\widehat{u}_{n}(t)\right]\right\|^{2} \geq \sum_{i=0}^{n} \lambda_{i}^{2 \alpha}\left(\alpha_{i}(t)-\alpha_{i}^{n}(t)\right)^{2} .
$$

Theorem 5.1. Let us suppose that propositions (H1)-(H4) are satisfied. Then we have the following:

(a) If $u_{0} \in D\left(A^{\alpha}\right)$, then for any $0<t_{0} \leq T_{0}$,

$$
\sup _{t_{0} \leq t \leq T_{0}}\left[\sum_{i=0}^{n} \lambda_{i}^{2 \alpha}\left(\alpha_{i}(t)-\alpha_{i}^{n}(t)\right)^{2}\right] \longrightarrow 0, \quad \text { as } n \rightarrow \infty .
$$

(b) If $u_{0} \in D(A)$, then

$$
\sup _{0 \leq t \leq T_{0}}\left[\sum_{i=0}^{n} \lambda_{i}^{2 \alpha}\left(\alpha_{i}(t)-\alpha_{i}^{n}(t)\right)^{2}\right] \longrightarrow 0, \quad \text { as } n \rightarrow \infty .
$$

As a consequence of Theorems 3.1 and 4.1, we have the following result.

Proposition 5.2. Let us suppose that assumptions (H1)-(H4) are satisfied. Then we have the following:

(a) If $u_{0} \in D\left(A^{\alpha}\right)$, then for any $0<t_{0} \leq T_{0}$,

$$
\left\|\widehat{u}_{n}-\widehat{u}_{m}\right\|_{T, \alpha} \longrightarrow 0, \quad \text { as } m, n \rightarrow \infty,
$$

i.e., $\widehat{u}_{n}$ is a cauchy sequence in $\mathcal{W}$ on $\left[t_{0}, T_{0}\right]$.

(b) If $u_{0} \in D(A)$, then

$$
\left\|\widehat{u}_{n}-\widehat{u}_{m}\right\|_{T, \alpha} \longrightarrow 0, \quad \text { as } m, n \rightarrow \infty,
$$

i.e., $\widehat{u}_{n}$ is a cauchy sequence in $\mathcal{W}$ on $\left[0, T_{0}\right]$.

Proof. Letting $n \geq m$ and $0 \leq \alpha<\vartheta$, we have

$$
\begin{aligned}
\left\|\widehat{u}_{n}(t)-\widehat{u}_{m}(t)\right\|_{\alpha} & =\left\|P^{n} u_{n}(t)-P^{m} u_{m}(t)\right\|_{\alpha} \\
& \leq\left\|P^{n}\left[u_{n}(t)-u_{m}(t)\right]\right\|_{\alpha}+\left\|\left(P^{n}-P^{m}\right) u_{m}\right\|_{\alpha} \\
& \leq\left\|u_{n}(t)-u_{m}(t)\right\|_{\alpha}+\frac{1}{\lambda_{m}^{\vartheta-\alpha}}\left\|A^{\vartheta} u_{m}\right\| .
\end{aligned}
$$


If $u_{0} \in D\left(A^{\alpha}\right)$, then the result in (a) follows from Theorem 4.1. If $u_{0} \in D(A)$, (b) follows from Corollary 4.2 .

For the convergence of $\widehat{u}_{n}(t) \rightarrow u(t)$, we have the following theorem.

Theorem 5.3. Let assumptions $(\mathrm{H} 1)-(\mathrm{H} 4)$ be satisfied, and let $u_{0} \in$ $D\left(A^{\alpha}\right)$ or $D(A)$. Then there exists a unique function $\widehat{u}_{n} \in \mathcal{W}$ satisfying

$$
\begin{aligned}
\widehat{u}_{n}(t)= & \int_{0}^{\infty} \xi_{\eta}(\theta) S\left(t^{\eta} \theta\right)\left[u(0)+g_{n}\left(0, u_{0}\right)\right] d \theta-g_{n}\left(t, \hat{u}_{n}(t)\right) \\
& +\eta \int_{0}^{t} \int_{0}^{\infty} \theta \xi_{\eta}(\theta)(t-s)^{\eta-1} A S\left((t-s)^{\eta} \theta\right) g_{n}\left(s, \hat{u}_{n}(s)\right) d \theta d s \\
& +\eta \int_{0}^{t} \int_{0}^{\infty} \theta \xi_{\eta}(\theta)(t-s)^{\eta-1} S\left((t-s)^{\eta} \theta\right) \\
& f_{n}\left(s, \widehat{u}_{n}(s), \hat{u}_{n}\left(h_{n}\left(\widehat{u}_{n}(s), s\right)\right)\right) d \theta d s, \quad t \in\left[0, T_{0}\right],
\end{aligned}
$$

and $u \in \mathcal{W}$

$$
\begin{aligned}
u(t)= & \int_{0}^{\infty} \xi_{\eta}(\theta) S\left(t^{\eta} \theta\right)\left[u(0)+g\left(0, u_{0}\right)\right] d \theta-g_{n}(t, \widehat{u}(t)) \\
& +\eta \int_{0}^{t} \int_{0}^{\infty} \theta \xi_{\eta}(\theta)(t-s)^{\eta-1} A S\left((t-s)^{\eta} \theta\right) g(s, u(s)) d \theta d s \\
& +\eta \int_{0}^{t} \int_{0}^{\infty} \theta \xi_{\eta}(\theta)(t-s)^{\eta-1} S\left((t-s)^{\eta} \theta\right) \\
& f(s, u(s), u(h(u(s), s))) d \theta d s, \quad t \in\left[0, T_{0}\right],
\end{aligned}
$$

such that $\widehat{u}_{n} \rightarrow u$ as $n \rightarrow \infty$ in $\mathcal{W}$ and $u$ satisfies (3.9) on $\left[0, T_{0}\right]$.

6. Example. We consider the following fractional order partial differential equation with a deviated argument:

$$
\left\{\begin{array}{l}
\partial_{t}^{\eta}\left[w(t, x)+\partial_{x} f_{1}(t, w(t, x))\right]-\partial_{x}^{2}[w(t, x)] \\
\quad=f_{2}(x, w(t, x))+f_{3}(t, x, w(t, x)), \quad x \in(0,1), t>0, \eta \in[0,1), \\
\\
w(t, 0)=w(t, 1)=0, \\
w(0, x)=u_{0}, \quad x \in(0,1),
\end{array}\right.
$$

where

$$
f_{2}(x, w(t, x))=\int_{0}^{x} K(x, s) w(s, k(t)|w(s, t)|) d s .
$$


The function $f_{3}: \mathbf{R}_{+} \times[0,1] \times \mathbf{R} \rightarrow \mathbf{R}$ is measurable in its second variable $x$, locally Hölder continuous in its first variable $t$, locally Lipschitz continuous in its third variable $w$ and uniformly in $x$. Further, we assume that $k: \mathbf{R}_{+} \rightarrow \mathbf{R}_{+}$is locally Hölder continuous in $t$ with $k(0)=0$ and $K(\cdot, \cdot) \in C^{1}([0,1] \times[0,1] ; \mathbf{R})$.

Let $X=L^{2}((0,1) ; \mathbf{R}), A u=d^{2} u / d x^{2}, D(A)=H^{2}(0,1) \cap H_{0}^{1}(0,1)$, $X_{1 / 2}=D\left((A)^{1 / 2}\right)=H_{0}^{1}(0,1)$ and $X_{-1 / 2}=\left(H_{0}^{1}(0,1)\right)^{*}=H^{-1}(0,1) \equiv$ $H^{1}(0,1)$. by

For $x \in(0,1)$, we define the function $f: \mathbf{R}_{+} \times X_{1 / 2} \times X_{-1 / 2} \rightarrow X$

$$
f(t, u, \xi)(x)=f_{2}(x, \xi)+f_{3}(t, x, u),
$$

where $f_{2}:[0,1] \times X \rightarrow H_{0}^{1}(0,1)$ is given by

$$
f_{2}(t, \xi)=\int_{0}^{x} K(x, y) \xi(y) d y,
$$

and $f_{3}: \mathbf{R} \times[0,1] \times H^{2}(0,1) \rightarrow H_{0}^{1}(0,1)$ satisfies the following

$$
\left\|f_{3}(t, x, u)\right\| \leq Q(x, t)\left(1+\|u\|_{H^{2}(0,1)}\right),
$$

with $Q(\cdot, t) \in X$ and $Q$ is continuous in its second argument. Next we assume that the function $h: H_{0}^{1}(0,1) \times \mathbf{R}_{+} \rightarrow \mathbf{R}_{+}$is defined by

$$
h(u(x, t), t)=k(t)|u(x, t)| .
$$

For $u \in D(A)$ and $\lambda \in \mathbf{R}$ with $-A u=\lambda u$, we have

$$
\begin{aligned}
\langle-A u, u\rangle & =\langle\lambda u, u\rangle \\
\left\|u^{\prime}\right\|_{L^{2}} & =\lambda\|u\|_{L^{2}},
\end{aligned}
$$

so we have $\lambda>0$. The solution $u$ of $-A u=\lambda u$ is

$$
u(x)=D_{1} \cos (\sqrt{\lambda} x)+D_{2} \sin (\sqrt{\lambda} x) .
$$

Using the boundary condition, we get $D_{1}=0$ and $\lambda=\lambda_{n}=n^{2} \pi^{2}$ for $n \in \mathbf{N}$. Thus, for $n \in \mathbf{N}$, we have

$$
u_{n}(x)=D_{2} \sin \left(\sqrt{\lambda_{n}} x\right) .
$$


Also $\left\langle u_{n}, u_{m}\right\rangle=0, m \neq n$ and $\left\langle u_{n}, u_{n}\right\rangle=1$. So, for $u \in D(A)$, there exists a sequence $\alpha_{n}$ of real numbers such that $u(x)=\sum_{n \in \mathbf{N}} \alpha_{n} u_{n}(x)$ with $\sum_{n \in \mathbf{N}}\left(\alpha_{n}\right)^{2}<\infty$ and $\sum_{n \in \mathbf{N}}\left(\alpha_{n}\right)^{2}\left(\lambda_{n}\right)^{2}<\infty$.

The semigroup is given by

$$
S(t) u=\sum_{n \in \mathbf{N}} \exp \left(-n^{2} t\right)\left\langle u, u_{m}\right\rangle u_{m} .
$$

The abstract formulation of (6.1) can be written as the following:

$$
\begin{aligned}
\frac{d^{\eta}}{d t^{\eta}}[u(t)+g(t, u(t))]+A u(t) & =f(t, u(t), u[h(u(t), t)]), \quad t>0, \eta[0,1), \\
(6.8) \quad u(0) & =u_{0},
\end{aligned}
$$

where $u(t)=w(t, \cdot)$ that is $u(t)(x)=w(t, x), x \in(0,1)$. The function $g: \mathbf{R}_{+} \times X_{1 / 2} \rightarrow X$, such that $g(t, u(t))(x)=\partial_{x} f_{1}(t, w(t, x))$.

It's not difficult to prove that all the assumptions $(\mathrm{H} 1)-(\mathrm{H} 4)$ are satisfied. For more details, see [7].

Acknowledgments. We highly appreciate the valuable comments and suggestions of the referees on our manuscript which helped to considerably improve the quality of the manuscript.

\section{REFERENCES}

1. D. Bahuguna and M. Muslim, A study of nonlocal history-valued retarded differential equations using analytic semigroups, Nonlin. Dyn. Syst. Theor. 6 (2006), $63-75$.

2. D. Bahuguna, S.K. Srivastava and S. Singh, Approximations of solutions to semilinear integrodifferential equations, Numer. Funct. Anal. Optim. 22 (2001), 487-504.

3. Norman W. Bazley, Approximation of wave equations with reproducing nonlinearities, Nonlin. Anal. 3 (1979), 539-546.

4. - Approximation of operators with reproducing nonlinearities, Manuscr. Math. 18 (1976), 353-369.

5. M. El-Borai, Some probability densities and fundamental solutions of fractional evolution equations, Chaos Solitons Fractals 14 (2002), 433-440.

6. L.E. El'sgol'ts and S.B. Norkin, Introduction to the theory and application of differential equations with deviating arguments, Math. Sci. Engineer. 105, Academic Press, New York, 1973.

7. C.G. Gal, Nonlinear abstract differential equations with deviated argument, J. Math. Anal. Appl. 333 (2007), 971-983. 
8. L.J. Grimm, Existence and uniqueness for nonlinear neutral-differential equations, Bull. Amer. Math. Soc. 77 (1971), 374-376.

9. Rajib Haloi, Dhirendra Bahuguna and Dwijendra N. Pandey, Existence and uniqueness of solutions for quasi-linear differential equations with deviating arguments, Electron. J. Diff. Eq. 13 (2012), 1-10.

10. J.T. Hoag and R.D. Driver, A delayed-advanced model for the electrodynamics two-body problem, Nonlin. Anal. 15 (1990), 165-184.

11. I..G. Klyuchnik and G.V. Zavīzion, On the asymptotic integration of a singularly perturbed system of linear differential equations with deviating arguments, translation in Nonlin. Oscil. 13 (2010), 178-195.

12. V. Lakshmikantham, S. Leela and Devi J. Vasundhara, Theory of fractional dynamic systems, Cambridge Scientific Publishers, Cambridge, 2009.

13. H. Lee, H. Alkahby and G. N'Guérékata, Stepanov-like almost automorphic solutions of semilinear evolution equations with deviated argument, Int. J. Evol. Eq. 3 (2008), 217-224.

14. F. Mainardi, On the initial value problem for the fractional diffusion-wave equation, Waves and stability in continuous media, 246-251, Ser. Adv. Math. Appl. Sci.23 (1994), 246-251.

15. _ On a special function arising in the time fractional diffusionwave equation, Transform methods and special functions, 171-183, Science Culture Technology, Singopore, 1995.

16. F. Mainardi and R. Gorenflo, On Mittag-Leffler-type functions in fractional evolution processes. Higher transcendental functions and their applications, J. Comput. Appl. Math. 118 (2000), 283-299.

17. F. Mainardi, A. Mura and G. Pagnini, The functions of the Wright type in fractional calculus, Lect. Notes Sem. Inter. Math. 09 (2010), 111-128.

18. The $M$-Wright function in time-fractional diffusion processes: $A$ tutorial survey, Int. J. Diff. Eq. 2010 (2010), 1-29.

19. M.W. Michalski, Derivatives of non-integer order and their applications, Disser. Math., Inst. Math. Pol. Acad. Sci., 1993.

20. P.D. Miletta, Approximation of solutions to evolution equations, Math. Meth. Appl. Sci. 17 (1994), 753-763.

21. K.S. Miller and B. Ross, An introduction to the fractional calculus and differential equations, John Wiley, New York, 1993.

22. M. Muslim, C. Carlos and A.K. Nandakumaran, Approximation of solutions to fractional integral equations, Comp. Math. Appl. 59 (2010), 1236-1244.

23. M. Muslim and A.K. Nandakumaran, Existence and approximations of solutions to some fractional order functional integral equations, J. Int. Eq. Appl. 22 (2010), 95-114.

24. S.K. Ntouyas and D. O'Regan, Existence results for semilinear neutral functional differential inclusions via analytic semigroups, Acta Appl. Math. 98 (2007), 223-253. 
25. R.J. Oberg, On the local existence of solutions of certain functionaldifferential equations, Proc. Amer. Math. Soc. 20 (1969), 295-302.

26. A. Pazy, Semigroups of linear operators and applications to partial differential equations, Springer-Verlag, New York, 1983.

27. I. Podlubny, Fractional differential equations, Academic Press, New York, 1999.

28. H. Pollard, The representation of $e^{-x^{\lambda}}$ as a Laplace integral, Bull. Amer. Math. Soc. 52 (1946), 908-910.

29. S. Stević, Bounded solutions of some systems of nonlinear functional differential equations with iterated deviating argument, Appl. Math. Comp. 218 (2012), 10429-10434.

30. J. Wang and Y. Zhou, Existence and controllability results for fractional semilinear differential inclusions, Nonlin. Anal. Real World Appl. 12 (2011), 36423653.

31. Rong N. Wang, De-H. Chen and Ti-J. Xiao, Abstract fractional Cauchy problems with almost sectorial operators, J. Diff. Eq. 252 (2012), 202-235.

32. L. Zhang, G. Wang and G. Song, Mixed boundary value problems for second order differential equations with different deviated arguments, J. Appl. Math. Inf. 29 (2011), 191-200.

33. Y. Zhou and F. Jiao, Nonlocal Cauchy problem for fractional evolution equations, Nonlin. Anal. Real World Appl. 11 (2010), 4465-4475.

Department of Mathematics and Statistics, Indian Institute of Technology Kanpur, India, Pin- 208016

Email address: prdipk@gmail.com

Department of Mathematics, Indian Institute of Technology Roorkee, INDIA, PIN- 247667

Email address: dwij.iitk@gmail.com, dwijpfma@iitr.ernet.in

Department of Mathematics and Statistics, Indian Institute of Technology

KANPUR, India, Pin- 208016

Email address: dhiren@iitk.ac.in 